\title{
Prostate cancer - Therapy with radium-223
}

\section{CÂncer de PRóstata - Terapia com RÁdio-223}

\author{
Authorship: Brazilian Society of Nuclear Medicine
}

Participants: Ana Emília Brito ${ }^{1}$, Bárbara Juarez Amorim², Milena Martello³, Wanderley Marques Bernardo ${ }^{3}$, Elba Etchebehere ${ }^{4}$

Final draft: December 11, 2017

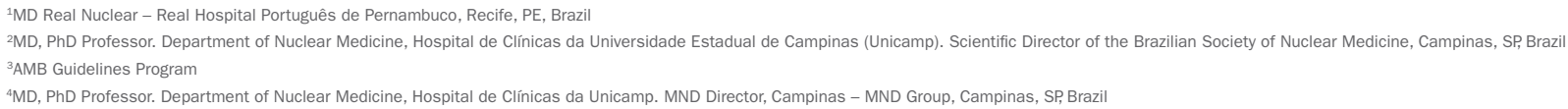

The Guidelines Project, an initiative of the Brazilian Medical Association, aims to combine information from the medical field in order to standardize procedures to assist the reasoning and decision-making of doctors.

The information provided through this project must be assessed and criticized by the physician responsible for the conduct that will be adopted, depending on the conditions and the clinical status of each patient.

\section{DESCRIPTION OF THE EVIDENCE COLLECTION METHOD}

This guideline followed the standard of a systematic review with evidence retrieval based on evidence-based medicine (EBM), so that clinical experience is integrated with the ability to critically analyze and apply scientific information rationally, thus improving the quality of medical care.

We used the structured mode of formulating questions synthesized by the acronym PICO, where P stands for patients with prostate cancer, I stands for indicator, i.e., radium-223, and O stands for outcome, which is benefit.

Based on the structured question, we identified the descriptors that formed the basis of the search for evidence in the databases: Medline-Pubmed. Ninety-nine (99) studies were retrieved and, after applying the eligibility criteria (inclusion and exclusion), 13 were selected to answer the clinical question (Annex I).

\section{Clinical question}

What is the benefit of radium-223 therapy in prostate cancer?

\section{Grades of ReCommendation AND LeVELS OF EVIDENCE}

- A: Experimental or observational studies of higher consistency.

- B: Experimental or observational studies of lower consistency.
- C: Cases reports / non-controlled studies.

- D: Opinion without critical evaluation, based on consensus, physiological studies or animal models.

\section{Овjective}

The aim of this guideline is to estimate the benefit of radium-223 chloride therapy ( $\mathrm{Ra} 223$ ) in patients with prostate cancer resistant to castration and bone metastases.

\section{INTRODUCTION}

Ra223 has been approved by the Brazilian Health Surveillance Agency (Anvisa) to be used as a drug in the treatment of patients with bone metastases from metastatic castration-resistant prostate cancer (mCRPC) without known visceral metastases. ${ }^{1}$

This treatment is indicated when patients with $\mathrm{mCRPC}$ have blastic bone metastases in an attempt to increase survival, but it is also applied in these conditions when patients have symptoms, such as pain (but not exclusively).

Ra223 is a low-range $(<100 \mu \mathrm{m})$, high-energy $(27.4$ $\mathrm{MeV}$ ) alpha particle emitter, similar to calcium, which is directed to areas where bone remodeling is increased, which occurs in sclerotic bone metastases of prostate cancer. The high energy of alpha particles causes the DNA to break down in the metastatic cells (cytotoxic effect), with low damage in the adjacent cells due to their low reach $(<10$ cells $){ }^{2}$ 


\section{Data extraction}

\section{Survival}

After evaluating $64 \mathrm{mCRPC}$ patients eligible for radiation therapy due to bone pain, with mean follow-up time of 18 months, there was greater survival among patients treated with Ra223 (50 kBq/kg dosage) compared to a placebo. Median survival was 65.3 versus 46.4 weeks, respectively, significantly higher for Ra223 $(50 \mathrm{kBq} / \mathrm{kg}$ dosage) $(\mathrm{p}=0.006)$ compared to a placebo $(\mathrm{HR}=2.12,95 \mathrm{CI}$ 1.12-3.98; $\mathrm{p}=0.020$ ), indicating a higher risk of death for the placebo group. ${ }^{3}$ (A) After 2 years of follow-up of these patients, the median survival of the Ra223-treated group was 71 weeks versus 46.4 weeks in the placebo group $\left(\mathrm{HR}=0.445\right.$, 95CI 0.232-0.851). ${ }^{4}(\mathbf{A})$

In the largest phase III multicenter controlled randomized trial evaluating $921 \mathrm{mCRPC}$ patients, 614 received six injections of Ra223 (50 kBq/Kg dosage) and 307 received placebo injections. Those patients treated with Ra223 had greater survival compared to those treated with placebo, with a median survival of 14.9 versus 11.3 months. The absolute increase in survival was 10.5 months (95CI 3.3-17.7). In this case, it is necessary to treat nine patients to have an increase in survival of 3.6 months (on average) in one patient. ${ }^{5}(\mathbf{A})$

When this same population was stratified according to prior use of docetaxel, Ra223 continued to show an increase in survival, both in the group of prior use $(\mathrm{HR}=0.70,95 \mathrm{CI}$ 0.56-0.88; $\mathrm{p}=0.002)$ compared to the group that had never used docetaxel $(\mathrm{HR}=0.69,95 \mathrm{CI} 0.52-0.92 ; \mathrm{p}=0.01) .{ }^{6}(\mathbf{A})$

\section{Bone event}

In an initial study with $64 \mathrm{mCRPC}$ patients, there was longer time until the first bone event in the Ra223-treated group compared to the placebo group (14 weeks versus 11 weeks) (HR=1.75, 95CI 0.96-3.19; $\mathrm{p}=0.065)$. However, there was no significant difference in the number of bone events $(\mathrm{p}=0.625){ }^{3}(\mathbf{B})$

In the large ALSYMPCA study, evaluating 921 patients with mCRPC, patients treated with Ra223 had longer time until the first bone event compared to the placebo group. Median time until the first bone event was 15.6 months with Ra223 versus 9.8 months with a placebo $(\mathrm{HR}=0.66,95 \mathrm{CI} 0.52-0.83 ; \mathrm{p}<0.001){ }^{5}(\mathbf{A})$

The data from the above study were further analyzed, focusing exclusively on the skeletal events of the mCRPC population. ${ }^{7}$ (A) The authors demonstrated that symptomatic skeletal events occurred in $33 \%$ of patients in the Ra223 group and 38\% in the placebo group. The Ra223 group required less use of radiotherapy to treat pain (HR=0.67, 95CI 0.53-0.85) and presented a lower spinal compression rate $(\mathrm{HR}=0.52,95 \mathrm{CI} 0.29-0.93)$. Despite this, the use of Ra223 did not significantly reduce the risk of occurrence of bone events $(\mathrm{HR}=0.62,95 \mathrm{CI}$ $0.35-1.09)$ nor the risk of surgical interventions $(\mathrm{HR}=0.72$, 95CI 0.28-1.82). ${ }^{7}$ (A)

Another study stratified the population described above according to prior use of docetaxel or not. 352 patients who used docetaxel prior to Ra223 were evaluated versus 262 patients who used only Ra223. There was a reduction in time until the first bone event only in the group of patients taking Ra223 who had previously used docetaxel $(\mathrm{p}=0.0009) \cdot{ }^{6}(\mathbf{A})$

\section{DOSE TOXICITY AND SAFETY}

In the initial randomized controlled multicenter phase II trial of 64 mCRPC, with patients divided into Ra223 and placebo groups, there was no significant difference in hematological toxicity. In addition, no patient discontinued treatment for this reason. ${ }^{3}(\mathbf{A})$

The use of different doses of Ra223 $(5 \mathrm{kBq} / \mathrm{kg}$ in 26 patients; $25 \mathrm{kBq} / \mathrm{kg}$ in 25 patients; $50 \mathrm{kBq} / \mathrm{kg}$ in $25 \mathrm{pa}$ tients; $100 \mathrm{kBq} / \mathrm{kg}$ in 24 patients) in $100 \mathrm{mCRPC}$ patients showed no difference in adverse effects among the groups analyzed, and $97 \%$ of the patients reported at least one adverse effect. Adverse effects included nausea, fatigue, vomiting, diarrhea, constipation, bone pain, urinary tract infection and peripheral edema. ${ }^{8}(\mathbf{A})$

Then, another study with a slightly larger casuistry (122 patients) was conducted to evaluate the efficacy and safety of different dosages of Ra223. The doses administered were $25 \mathrm{kBq} / \mathrm{kg}$ in 41 patients, $50 \mathrm{kBq} / \mathrm{kg}$ in 39 patients and $80 \mathrm{kBq} / \mathrm{kg}$ in 42 patients, each of them undergoing a protocol of three applications every 4 weeks. The study demonstrated that dosages up to $80 \mathrm{kBq} / \mathrm{Kg}$ are safe. Ninety-two per cent $(92 \%)$ of the patients had at least one adverse effect: diarrhea (21\%), nausea (16\%) and anemia (14\%). There were no differences among the groups regarding survival, bone events, pain reduction or hematological events. ${ }^{9}(\mathbf{A})$

In the ALSYMPCA study (N=921), the overall number of adverse and hematological effects was lower in patients treated with Ra223 (93\%) compared to placebo (96\%), with $47 \%$ serious events in the first and $60 \%$ in the latter group. The number of grade 3 or 4 adverse hematological effects was not significantly higher in the group treated with $\operatorname{Ra} 223 .{ }^{5}(\mathbf{A})$

Subgroup analysis of ALSYMPCA patients stratified by prior docetaxel use showed that the incidence of grade 3 or 4 anemia and neutropenia was similar between Ra223 and placebo, regardless of previous use of docetaxel. ${ }^{6}(\mathbf{A})$ 
However, previous use of docetaxel led to a greater number of hematological adverse events in both Ra223 and placebo patients. However, grade 3 and 4 myelosuppression rates were low, with differences only in thrombocytopenia rates. In addition, previous use of docetaxel did not influence the number of non-hematological events. ${ }^{6}(\mathbf{A})$

The main non-hematological adverse events reported in the studies were diarrhea, constipation, vomiting, nausea, fatigue, bone pain and peripheral edema. ${ }^{3,5,6,9,10}(\mathbf{A})$

\section{Markers}

Evaluating the different dosages of Ra223 (25, 50 and 80 $\mathrm{kBq} / \mathrm{kg}$ ) in a total of 122 patients, there was a better alkaline phosphatase (AP) and prostate specific antigen (PSA) response in the groups receiving the highest doses (50 and $80 \mathrm{kBq} / \mathrm{kg}$ ). No patient in the $25 \mathrm{kBq} / \mathrm{kg}$ group achieved a $50 \%$ reduction in PSA. ${ }^{9}(\mathrm{~A})$

In the ALSYMPCA study, there was a significant decline in $\mathrm{AP}$ and a longer time interval for elevation of this marker in patients treated with Ra223 compared to placebo. There was also a significant reduction of PSA in the Ra223 (16\%) versus placebo (6\%) group, in addition to a longer time interval to raise this parameter. ${ }^{5}(\mathbf{A})$

In both studies described above, the AP and PSA values were analyzed after 12 weeks and the reduction was considered significant if greater than $30 \%$ of the initial value. This longer time to increase AP and PSA also occurred in the Ra223 group compared to placebo, regardless of previous docetaxel use $(\mathrm{p}<0.05) \cdot{ }^{6}(\mathbf{A})$

\section{IMPROVEMENT OF PAIN AND QUALITY OF LIFE}

A study with $100 \mathrm{mCRPC}$ patients aimed at evaluating different single doses of Ra223 (5, 25, 50 and $100 \mathrm{kBq} / \mathrm{kg}$ ) and had as primary outcome improvement of pain within 16 weeks after treatment. In this study, the groups receiving the highest Ra223 dosages ( 50 and $100 \mathrm{KBq} / \mathrm{Kg}$ ) required less of other forms of analgesia for pain control than the groups receiving lower Ra223 dosages (5 and $25 \mathrm{kBq} / \mathrm{kg}){ }^{8}(\mathbf{A})$

In the analysis of pain reduction alone among the different doses of Ra223 (25, 50 and $80 \mathrm{kBq} / \mathrm{kg}$ ) given to 122 patients, there was a tendency for better results using $50 \mathrm{kBq} / \mathrm{kg} .{ }^{9}$ (A) Despite the dose-response effect on pain reduction, there is no such analysis compared to placebo.

A subgroup analysis of patients from the ALSYMPCA ${ }^{5}$ study evaluated the improvement of quality of life. More patients treated with Ra223 showed improvement in quality of life assessment tests compared to patients treated with placebo $(29.2 \%$ versus $18.5 \%$; $\mathrm{p}=0.004) .{ }^{10}(\mathbf{A})$

\section{Hospitalization Rate}

Another study evaluated the hospitalization rate within the 12 months following treatment with Ra223 compared to placebo-treated patients. Patients receiving Ra223 had a lower hospitalization rate than those treated with placebo ( $37 \%$ and $45.5 \%$, respectively), regardless of whether a skeletal event occurred. In addition, the number of days of hospitalization in the Ra223 group was lower than in the placebo group (4.4 versus 6.6 days; $\mathrm{p}=0.004) .{ }^{11}(\mathbf{A})$

\section{Evidence SUMmary}

We were able to perform a meta-analysis of two outcomes studied: survival and bone event. The other outcomes could not be investigated by meta-analysis due to the lack of necessary data or use of the same population in different studies.

\section{SURVIVAL}

Two studies ${ }^{4,5}(\mathbf{A})$ totaling 985 patients (647 in the Ra223 group and 338 in the placebo group) were included in this analysis. In this comparison, at $0 \%$ heterogeneity, there was a $10 \%$ decline in mortality (95CI 4-16) in favor of Ra223 treatment. ${ }^{12}$ (A) Thus, 10 patients need to be treated to avoid one death compared to no treatment (Figure 1).

\section{BONE EVENTS}

Two studies were included in the analysis ${ }^{3,7}(\mathbf{A})$ totaling 985 patients (647 treated with radium-223 and 338 with a placebo). In this comparison there is a non-significant reduction in the risk of bone events of $5 \%$ (95CI -1-11) in favor of treatment with Ra223 and heterogeneity is $0 \%$. This means that there is no significant difference $(p<0.05)$ in the risk of bone events when comparing treatment and non-treatment with radio-223 (Figure 2).

\section{Recommendation}

Ra223 is effective for the treatment of patients with $\mathrm{mCRPC}$, with a reduction in mortality of $10 \%$ and an increase in mean survival over 3 months. There is no reduction in the number of bone events in these patients and no improvement in pain was observed except for the dose-response aspect.

Other benefits have been demonstrated, such as improved quality of life, increased time to skeletal events, reduced hospitalization and effect on markers such as PSA and AP.

The most common adverse events are both hematologic (anemia, neutropenia and thrombocytopenia) and non-hematological (diarrhea, constipation, vomiting, nausea, fatigue, bone pain and peripheral edema). 


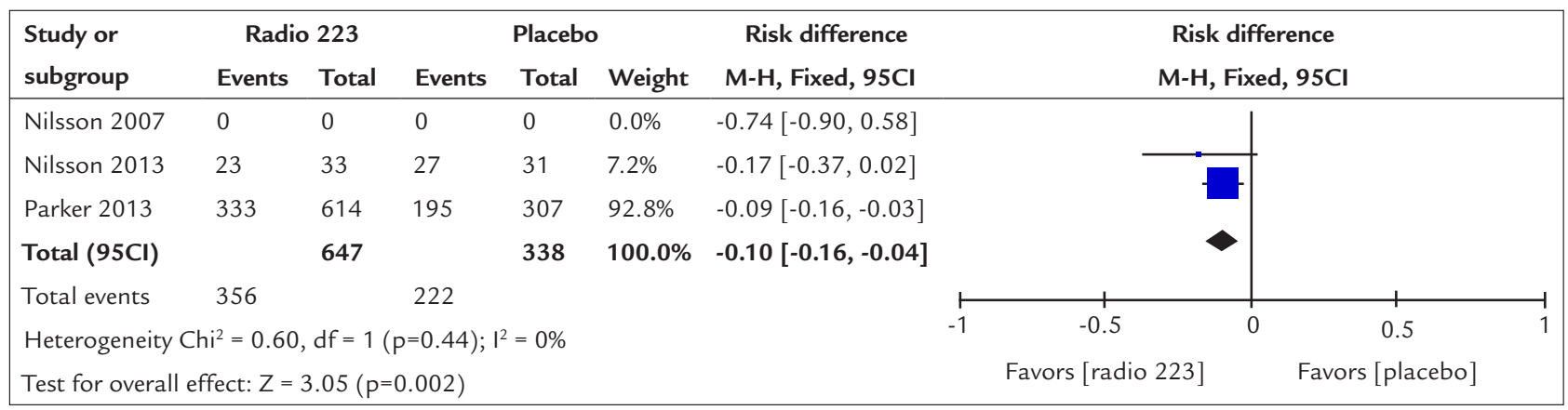

FIGURE 1 Summary of the studies comparing the use of Ra223 versus placebo in the treatment of mCRPC regarding the outcome of increased survival.

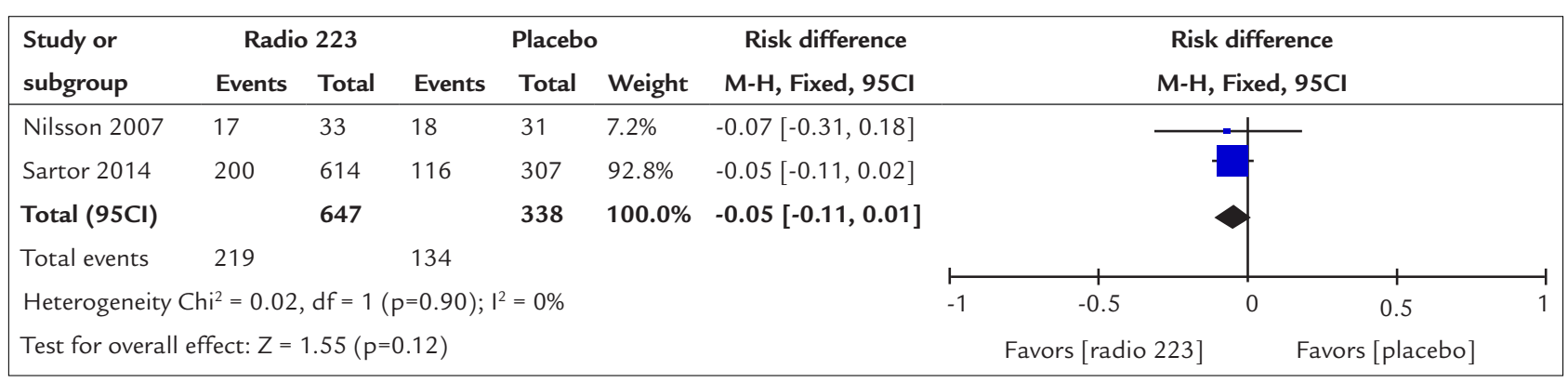

FIGURE 2 Summary of the studies comparing the use of Ra223 versus placebo in the treatment of mCRPC regarding the outcome of number of bone events.

Treatment with six doses of $55 \mathrm{kBq} / \mathrm{Kg}$ of intravenous $\mathrm{Ra} 223$ injections every 30 days is recommended for patients with mCRPC and bone metastases.

\section{Discussion AND PERSPeCtives}

At the moment only five other medications, in addition to Ra223, which produce a demonstrated increase in survival in patients with mCRPC (docetaxel, cabazitaxel, abiraterone, enzalutamide, sipuleucel-T) are available. In view of this scenario, Ra223 stands out as a treatment with few contraindications and acceptable adverse effects, and an excellent option for mCRPC patients.

Although the studies presented here use a dose of 50 $\mathrm{kBq} / \mathrm{kg}$, the commercial dose was adjusted to $55 \mathrm{kBq} / \mathrm{kg}$ to meet the standardization criteria. ${ }^{13}(\mathbf{D})$

Only one study carried out re-treatment with Ra223 in mCRPC patients. ${ }^{14}$ (B) Although it is a possibility, since the study showed safety, we do not recommend repeating the treatment until further studies are performed.

Studies are being conducted to validate the concomitant use of Ra223 with other therapies. We highlight the combination of Ra223 treatment with enzalutamide (phase III studies), abiraterone (phase II: NCT02097303), denosumab (phase II: NCT02366130), bicalutamide (phase II:
NCT02582749) and radiotherapy (phase II: NCT02484339). In addition, studies in asymptomatic patients are being performed (NCT03002220).

Ra223 is also being studied to treat other diseases such as osteosarcoma (NCT01833520), multiple myeloma (NCT02928029) and breast cancer (phase II: NCT02258451).

As soon as these studies are available, we will update this guideline.

\section{Conflict of interest}

The authors state that there is no conflict of interest regarding this review.

\section{REFEREnCES}

1. ANVISA. Registro ANVISA n ${ }^{\circ} 170560104$ - XOFIGO [cited 2017 Apr 9]. Available from: https://www.smerp.com.br/anvisa/?ac=prodDetail\&anvisa Id $=170560104$

2. Nilsson S. Radium-223 therapy of bone metastases in prostate cancer. Semin Nucl Med. 2016; 46(6):544-56.

3. Nilsson S, Franzén L, Parker C, Tyrrell C, Blom R, Tennvall J, et al. Bonetargeted radium-223 in symptomatic, hormone-refractory prostate cancer: a randomised, multicentre, placebo-controlled phase II study. Lancet Oncol. 2007; 8(7):587-94.

4. Nilsson S, Franz L, Parker C, Tyrrell C, Blom R, Tennvall J, et al. Two-year survival follow-up of the randomized, double-blind, placebo-controlled phase II study of radium-223 chloride in patients with castration-resistant prostate cancer and bone metastases. Clin Genitourin Cancer. 2013; 11(1):20-6. 
5. Parker C, Nilsson S, Heinrich D, Helle SI, O'Sullivan JM, Fosså SD, et al.; ALSYMPCA Investigators. Alpha emitter radium-223 and survival in metastatic prostate cancer. N Engl J Med. 2013; 369(3):213-23.

6. Hoskin P, Sartor O, O'Sullivan JM, Johannessen DC, Helle SI, Logue J, et al. Efficacy and safety of radium-223 dichloride in patients with castrationresistant prostate cancer and symptomatic bone metastases, with or without previous docetaxel use: a prespecified subgroup analysis from the randomised, double-blind, phase 3 ALSYMPC trial. Lancet Oncol. 2014; 15(12):1397-406.

7. Sartor O, Coleman R, Nilsson S, Heinrich D, Helle SI, O'Sullivan JM, et al. Effect of radium-223 dichloride on symptomatic skeletal events in patients with castration-resistant prostate cancer and bone metastases: Results from a phase 3, double-blind, randomised trial. Lancet Oncol. 2014; 15(7):738-46.

8. Nilsson S, Strang P, Aksnes AK, Franzèn L, Olivier P, Pecking A, et al. A randomized, dose-response, multicenter phase II study of radium-223 chloride for the palliation of painful bone metastases in patients with castration-resistant prostate cancer. Eur J Cancer. 2012; 48(5):678-86.

9. Parker CC, Pascoe S, Chodacki A, O'Sullivan JM, Germá JR, O'BryanTear CG, et al. A randomized, double-blind, dose-finding, multicenter, phase 2 study of radium chloride ( $R$ a 223) in patients with bone metastases and castration-resistant prostate cancer. Eur Urol. 2013; 63(2):189-97.

10. Nilsson S, Cislo P, Sartor O, Vogelzang NJ, Coleman RE, O'Sullivan JM, et al. Patient-reported quality-of-life analysis of radium-223 dichloride from the phase III ALSYMPCA study. Ann Oncol. 2016; 27(5):868-74.

11. Parker C, Zhan L, Cislo P, Reuning-Scherer J, Vogelzang NJ, Nilsson S, et al. Effect of radium-223 dichloride (Ra-223) on hospitalisation: an analysis from the phase 3 randomised Alpharadin in Symptomatic Prostate Cancer Patients (ALSYMPCA) trial. Eur J Cancer. 2017; 71:1-6.

12. Laupacis A, Sackett DL, Roberts RS. An assessment of clinically useful measures of the consequences of treatment. N Engl J Med. 1988; 318(26):1728-33.

13. U.S.NRC. Office of Nuclear Material Safety and Safeguards. 2010; 1:1-6.

14. Radium-223 (Ra-223) Re-treatment (Re-tx): first experience from an international, multicenter, prospective study in patients (Pts) with castration-resistant prostate cancer and bone metastases (mCRPC). Clin Adv Hematol Oncol. 2016; 14(4 Suppl 5):10-1.

15. Jadad AR, Moore RA, Carroll D, Jenkinson C, Reynolds DJ, Gavaghan DJ, et al. Assessing the quality of reports of randomized clinical trials: is blinding necessary? Control Clin Trials. 1996; 17(1):1-12.

16. Levels of Evidence and Grades of Recommendations - Oxford Centre for Evidence Based Medicine. Available from: http://cebm.jr2.ox.ac.uk/docs/old_levels.htm.

17. Goldet G, Howick J. Understanding GRADE: an introduction. J Evid Based Med. 2013; 6(1):50-4.

\section{AnNex I}

\section{Structured question}

- $\mathbf{P}$ - Patients with prostate cancer resistant to castration and bone metastases.

- I - Intravenous therapy with radium-223.

- C - Placebo.

- $\mathbf{O}$ - Benefit or harm.

\section{Search strategy}

PubMed-Medline

- \#1 - (Prostate Neoplasms OR Prostate Neoplasm OR Prostatic Neoplasm OR Prostate Cancer OR Prostate Cancers OR Prostatic Cancer OR Prostatic Cancers)

- \#2 - (radium OR Xofigo OR Ra 223)

- \#3 - random*

- 1st RETRIEVAL = \#1 AND \#2 AND \#3 = 99

\section{Articles retrieved}

Ninety-nine (99) articles were retrieved. Of these, 19 were selected by title and 11 by summary. After a critical analysis by three nuclear physicians, 13 studies were selected, using as inclusion criteria randomized clinical trials, greater strength of evidence and outcomes pertinent to the clinical doubt in question. The reason for excluding the other texts was that they were not randomized studies.

The scientific database consulted was Medline via Pubmed. A manual search was performed based on references of the reviews (narrative or systematic), as well as the selected studies.

\section{Inclusion criteria for selected studies}

Increased survival was the main outcome analyzed in this guideline; however, during its elaboration, it was possible to evaluate other outcomes, which are also presented.

\section{According to study design}

The studies included in this guideline were classified according to the Jadad score.$^{15}$ According to this classification, studies with Jadad less than three are inconsistent, while those with a score greater than three are considered consistent.

\section{Language}

We included studies available in Portuguese, English or Spanish.

\section{According to publication}

Only full-text studies were considered for critical assessment.

\section{Exposure of results}

For results with available evidence, the population, intervention, outcomes, presence or absence of benefit and/or harm and controversies will be defined in a specific manner, whenever possible.

\section{Recommendation}

The recommendations will be elaborated by the authors of the review, with the initial characteristic of synthesis of the evidence, and later validated by all the authors who participate in the elaboration of this Guideline.

The grade of recommendation stems directly from the available strength of included studies, according to the Oxford scale, ${ }^{16}$ and the GRADE system. ${ }^{17}$ 